\title{
Espacio fiscal para salud en Honduras
}

\author{
Lorena Prieto Toledo', Vilma Montañez Ginocchio ${ }^{1}$ y Camilo Cid-Pedraza ${ }^{2}$
}

Forma de citar Prieto Toledo L, Montañez Ginocchio V, Cid-Pedraza C. Espacio fiscal para salud en Honduras. Rev Panam Salud Publica. 2018;42:e8. https:/ / doi.org/10.26633/RPSP.2018.8

RESUMEN Objetivo. Analizar las fuentes de espacio fiscal para el sector salud en Honduras en un contexto de reforma del sector, con el compromiso de alcanzar una meta de gasto público en salud de 6\% del producto interno bruto (PIB).

Métodos. Se realizó un análisis de la condición básica y las fuentes de espacio fiscal en base a una revisión bibliográfica y datos secundarios. Se estimó el tamaño de las fuentes con datos oficiales, estadísticas internacionales y estudios previos. De manera complementaria al estudio, se realizó un análisis de la factibilidad política y se aplicó una encuesta en línea a actores clave. Resultados. Las estimaciones de la condición básica de crecimiento económico muestran que es necesario identificar otras fuentes para poder generar nuevos recursos. La reciente reforma tributaria limita la factibilidad política de generar nuevos impuestos, a excepción de los impuestos al pecado cuya recaudación se podría asignar exclusivamente a salud. La reforma de protección social abre el camino para explorar medidas que liberen recursos con mejoras en la eficiencia del sector. Una limitante en el caso del gasto público proveniente de la seguridad social es el techo de la base contributiva, independiente de una aceleración en la formalización laboral.

Conclusiones. Honduras puede avanzar en lograr la meta de un gasto público en salud de 6\% del PIB que respalde los planes de reforma sectorial, pero sus opciones se ven limitadas por la reciente reforma tributaria. La reforma de protección social en salud debe considerar los recursos adicionales que tendrá disponibles para no poner en riesgo su implementación.

Palabras clave Financiación de la atención de la salud; cobertura universal; América Latina; Honduras.

Honduras está en un proceso de reforma de su sector salud que se inició con la aprobación del nuevo Modelo Nacional de Salud (2013) y la Ley Marco de Protección Social (2015). Tiene una población de 7,96 millones de personas, de las cuales $45,9 \%$ vivía en áreas rurales en el año 2014 (1). Los índices de pobreza se han mantenido altos y alcanzaron $62,8 \%$ en 2014 (1). No obstante, ha logrado avances importantes en los indicadores de salud tales como las tasas de mortalidad neonatal, infantil y en menores de 5 años, y

\footnotetext{
Universidad del Pacífico de Perú, Lima, Perú. Enviar la correspondencia a Lorena Prieto Toledo, a.prietotoledo@up.edu.pe

2 Organización Panamericana de la Salud, Washington DC, Estados Unidos de América.
}

la razón de mortalidad materna y alcanzó una esperanza de vida al nacer de 74 años en el año 2013 (1). Estos resultados se sustentan, en parte, en las mejoras en la cobertura de los servicios de salud de la población (2).

El sistema de salud es mixto, segmentado y fragmentado. El sector público de salud se divide entre Secretaría de Salud (SESAL) y el Instituto Hondureño de Seguridad Social (IHSS), que constituyen fondos separados y coexisten con un sector privado pequeño. La SESAL se financia vía impuestos y es abierto a cualquier persona. El IHSS se financia con contribuciones y mantiene una red de provisión cerrada para sus beneficiarios (3-6) y cubre al $20 \%$ de la población.
El gasto total per cápita en salud alcanzó 400 dólares internacionales (\$int) ajustados por paridad de poder adquisitivo (PPA), o un gasto total en salud de $8,7 \%$ del PIB, en el año 2014 (1). El gasto de bolsillo es la principal fuente y representa $45,6 \%$ del gasto total en salud, mientras que el gasto de SESAL representa $35,5 \%$ e IHSS $12,5 \%(1,7)$.

Según el Fondo Monetario Internacional (FMI), sus perspectivas económicas son positivas según los acuerdos con el gobierno (8). En el 2014, el PIB creció $3,1 \%$, sus remesas del exterior representaron $15,8 \%$ del PIB, los ingresos tributarios fueron $16,5 \%$ del PIB y el déficit fiscal fue de $4,2 \%$ del PIB $(1,9)$.

Este es un artículo de acceso abierto distribuido bajo los términos de la licencia Creative Commons Attribution-NonCommercial-NoDerivs 3.0 IGO, que permite su uso, distribución y reproducción en cualquier medio, siempre que el trabajo original se cite de la manera adecuada. No se permiten modificaciones a los artículos ni su uso comercial. Al reproducir un artículo no debe haber ningún indicio de que la OPS o el artículo avalan a una organizaciôn un producto específico. El uso del logo de la OPS no está permitido. Esta leyenda debe conservarse, junto con la URL original del artículo. 
Honduras se ha comprometido con la estrategia regional para la cobertura y el acceso universal en salud de la Organización Panamericana de la Salud (OPS) y con llevar el gasto público en salud a $6 \%$ de PIB (10). El objetivo de este estudio es analizar las fuentes de espacio fiscal (EF) para salud en Honduras en un contexto de reforma tributaria reciente, un sistema segmentado y una reforma en el sector salud inminente.

\section{MATERIALES Y MÉTODOS}

Este es un estudio observacional longitudinal con información secundaria y revisión de la literatura. Se estima el EF como recursos adicionales para el sector público, sin poner en riesgo su sostenibilidad financiera (11). Según la literatura $(11,12)$ la condición básica para crear EF es la estabilidad en el crecimiento económico. Se identifican seis fuentes de EF: 1) generar nuevos ingresos, 2) repriorizar el gasto, 3) aumentar la eficiencia, 4) aumentar la deuda, 5) aumentar la ayuda externa y 6) la expansión monetaria. Aunque cada fuente se revisa según sus límites o los posibles efectos no deseados que podrían generar, la opción de expansión monetaria se descarta por sus implicancias inflacionarias (11).

Se estima el crecimiento económico con la elasticidad gasto-ingreso (E) para una serie larga de años, según la siguiente ecuación $(13,14)$ :

$$
E=\frac{\Delta \% \text { Gasto }}{\Delta \% P I B}
$$

La elasticidad anual se calcula para el gasto público en salud (GPS) - que incluye SESAL e IHSS-, el de SESAL (GPPS) y el de IHSS (GSSS) en tres escenarios:

- Escenario pesimista: descarta las elasticidades mayores a la elasticidad promedio más una desviación estándar.

- Escenario neutro: descarta las menores a la elasticidad promedio menos una desviación estándar y mayores al promedio más una desviación estándar.

- Escenario optimista: descarta las menores a la elasticidad promedio menos una desviación estándar.

Se utiliza la serie del PIB del Banco Mundial (1) para el período 1960-2014 y las proyecciones publicadas por el FMI (9) para el período 2015-2020.
Los nuevos ingresos para SESAL se estiman como cambios en la recaudación de impuestos; el análisis se centra en el impacto de la reforma tributaria del 2014 y en dos proyecciones de cambios en impuestos indirectos asociados a productos específicos. Estos implican riesgos en salud y en la literatura se denominan "sin taxes" ("impuestos al pecado"), según datos de la Secretaría de Finanzas (15-19). La primera proyección considera el impuesto existente a gaseosas (también llamadas agua o bebida gaseosa, bebida sin alcohol gasificada, bebida gaseosa o carbonatada, etc.), con un escenario base que proyecta el crecimiento de $0,31 \%$ anual de los últimos dos años en las ventas (en miles de litros) con el impuesto actual. En un segundo escenario, se considera un aumento de $10 \%$ del impuesto proyecta el crecimiento de $-0,04 \%$ anual del último año en las ventas (en miles de litros) (15-19). La segunda proyección considera el escenario de crear un impuesto nuevo a las comidas procesadas que genere una recaudación igual a las de gaseosas ${ }^{3}$. En las simulaciones de posibilidades de espacio fiscal, son considerados como impuestos "marcados", destinados en forma exclusiva para salud.

Los nuevos ingresos para IHSS consideran la estructura de las contribuciones (tasa, base, y techos). En la actualidad, el Estado contribuye (solo para trabajadores públicos) con $0,5 \%$, el empleador con $5,0 \%$, y el trabajador con $2,5 \%$ del salario, con un techo en la base contributiva de 7000 lempiras hondureñas (HNL) en el 2013, que aumentó a HNL 7350 para el 2014 y 2015 (335 dólares) (1)4 . La reforma mantiene las tasas, pero establece un aumento con un techo de 5\% anual. Se proyectan cuatro escenarios: 1) sin la gradualidad en el techo y se mantiene el crecimiento de $2,7 \%$ del número de cotizantes al año reportado durante el período 2010-2014, según datos del IHSS (20); 2) se simula y proyecta la gradualidad en el aumento del techo; 3) se acelera la formalización laboral de $2,7 \%$ a $4,1 \%$; y 4 ) se acelera la formalización a $8,7 \%$ para generar un aumento de un punto porcentual (pp) del PIB.

Las estimaciones de las otras fuentes se hacen en base a otros estudios $(11,21,22)$.

\footnotetext{
Estos cálculos no consideran la elasticidad de la demanda de estos productos.

4 Tipo de cambio oficial 21,945 HNL por USD en 2015.
}

El análisis de factibilidad política se realizó con dos metodologías: la revisión de acuerdos políticos, con agentes clave y la discusión pública reciente, y con la aplicación de una encuesta en línea voluntaria enviada a 31 actores clave del sector seleccionados por autoridades de SESAL, de los cuales 11 contestaron.

El alcance de este análisis de EF está en identificar posibles fuentes de nuevos recursos.

\section{RESULTADOS}

\section{Condición básica: crecimiento económico}

La evolución del GPS, GPPS y GSSS muestra que las fluctuaciones del GPS y GPPS son muy similares, pero no así el caso del GSSS (figura 1).

Incluidos todos los años de la serie, la elasticidad ingreso es de 1,069 del GPS, de 0,885 del GPPS y de 1,981 del GSSS. Así, aunque el GPS aumenta en 1,069\% con un aumento de $1 \%$ de PIB, el GPPS solo aumenta en $0,885 \%$. El GPS tiene una elasticidad de 0,307 en el escenario pesimista lo que se traduce en una caída del GPS como porcentaje del PIB en el año 2015, y una ligera alza en el resto del período (cuadro 1). En el escenario neutral, también muestra una caída inicial, mientras que en el escenario optimista el GPS aumenta en todos los años.

Las proyecciones son muy diferentes cuando se separan los fondos (cuadro 1). El GPPS muestra disminuciones en el caso pesimista y neutral. El escenario optimista solo muestra un aumento promedio anual de 0,0142 puntos porcentuales (pp), que llevaría el GPPS a 3,413\% del PIB al año 2020. El GSSS tiene elasticidades más elevadas y todos los escenarios generan aumentos en el fondo de IHSS. El escenario pesimista lleva a un aumento promedio anual 0,0097 pp del PIB, el neutral a 0,0138 y el optimista a 0,0230 .

Como las proyecciones del FMI ya incluyen la reforma tributaria y la nueva política fiscal, es necesario implementar acciones más allá de políticas macroeconómicas para generar EF.

\section{Nuevos ingresos: impuestos}

La principal fuente de recursos públi$\cos$ es la recaudación de impuestos, seguido por las donaciones y otros ingresos (23). Con la reforma tributaria del año 2014, la proyección de los ingresos 
FIGURA 1. GPS, GPPS y GSSS como porcentaje del PIB, 1996-2014

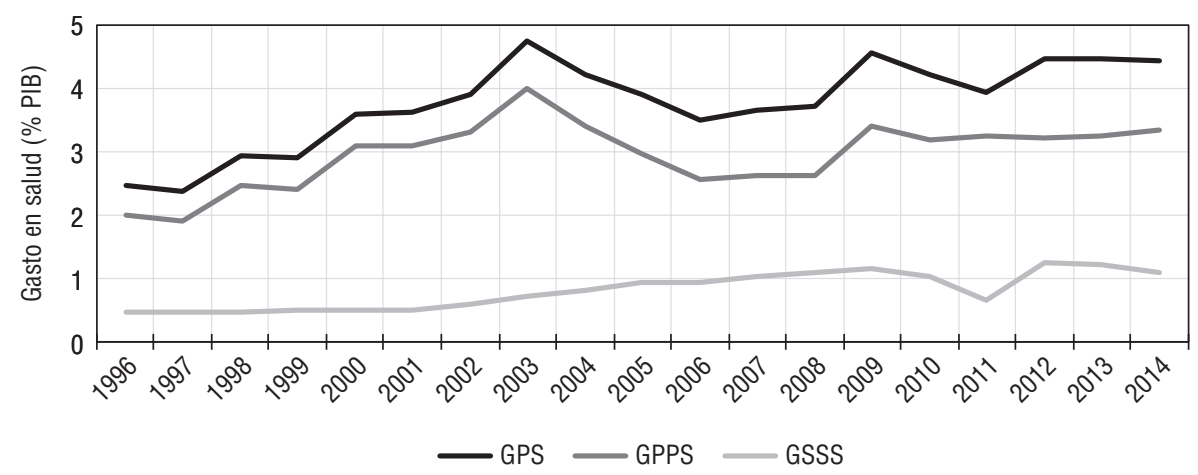

Elaboración propia

Fuente: Banco Mundial.

GPS, gasto público en salud; GPPS, gasto público en salud de la Secretaría de Salud; GSSS, gasto público en salud del Instituto Hondureño de Seguridad Social.

Cuadro 1. Elasticidades ingreso del gasto en salud, gasto en salud como porcentaje del producto interno bruto, cambio en puntos porcentuales por año, espacio fiscal promedio anual y acumulado

\begin{tabular}{|c|c|c|c|c|c|c|c|c|c|}
\hline & Elasticidad & 2015 & 2016 & 2017 & 2018 & 2019 & 2020 & Promedio & Acumulado \\
\hline \multicolumn{10}{|l|}{$\begin{array}{l}\text { GPS } \\
\text { Pesimista }\end{array}$} \\
\hline GPS (\% PIB) & 0,307 & 4,314 & 4,323 & 4,333 & 4,343 & 4,350 & 4,364 & & \\
\hline$\Delta \mathrm{pp}$ & & $-0,104$ & 0,009 & 0,009 & 0,010 & 0,007 & 0,014 & $-0,0090$ & $-0,054$ \\
\hline \multicolumn{10}{|l|}{ Neutro } \\
\hline GPS (\% PIB) & 1,082 & 4,430 & 4,443 & 4,456 & 4,469 & 4,483 & 4,498 & & \\
\hline $\begin{array}{l}\Delta p p \\
\text { Optimista }\end{array}$ & & 0,012 & 0,013 & 0,013 & 0,013 & 0,014 & 0,014 & 0,0133 & 0,080 \\
\hline GPS (\% PIB) & 1,843 & 4,544 & 4,560 & 4,576 & 4,593 & 4,614 & 4,629 & & \\
\hline$\Delta p p$ & & 0,126 & 0,016 & 0,017 & 0,017 & 0,021 & 0,015 & 0,0351 & 0,211 \\
\hline \multicolumn{10}{|l|}{$\begin{array}{l}\text { GPPS } \\
\text { Pesimista }\end{array}$} \\
\hline GPPS (\% PIB) & $-0,028$ & 3,212 & 3,204 & 3,197 & 3,189 & 3,178 & 3,173 & & \\
\hline$\Delta \mathrm{pp}$ & & $-0,116$ & $-0,008$ & $-0,008$ & $-0,008$ & $-0,011$ & $-0,005$ & $-0,0259$ & $-0,155$ \\
\hline \multicolumn{10}{|l|}{ Neutro } \\
\hline GPPS (\% PIB) & 0,959 & 3,323 & 3,318 & 3,314 & 3,309 & 3,303 & 3,298 & & \\
\hline \multicolumn{10}{|l|}{ Optimista } \\
\hline GPPS (\% PIB) & 1,864 & 3,425 & 3,423 & 3,421 & 3,418 & 3,418 & 3,413 & & \\
\hline$\Delta p p$ & & 0,097 & $-0,002$ & $-0,002$ & $-0,002$ & 0,000 & $-0,005$ & 0,0142 & 0,085 \\
\hline \multicolumn{10}{|l|}{$\begin{array}{l}\text { GSSS } \\
\text { Pesimista }\end{array}$} \\
\hline GSSS (\% PIB) & 0,781 & 1,082 & 1,094 & 1,107 & 1,120 & 1,133 & 1,148 & & \\
\hline \multicolumn{10}{|l|}{ Neutro } \\
\hline GSSS (\% PIB) & 1,339 & 1,102 & 1,115 & 1,129 & 1,143 & 1,158 & 1,173 & & \\
\hline $\begin{array}{l}\Delta p p \\
\text { Optimista }\end{array}$ & & 0,013 & 0,013 & 0,014 & 0,014 & 0,015 & 0,015 & 0,0138 & 0,083 \\
\hline GSSS (\% PIB) & 2,574 & 1,148 & 1,163 & 1,178 & 1,194 & 1,212 & 1,228 & & \\
\hline$\Delta p p$ & & 0,058 & 0,015 & 0,015 & 0,016 & 0,018 & 0,016 & 0,0230 & 0,138 \\
\hline
\end{tabular}

Elaboración propia.

Fuente: Banco Mundial (1), Organización Mundial de la Salud (7), Fondo Monetario Internacional (9).

GPS, gasto público en salud; GPPS, gasto público en salud de la Secretaría de Salud; GSSS, gasto público en salud del Instituto Hondureño de Seguridad Social; PIB, producto interno bruto; pp, puntos porcentuales.

públicos hasta el año 2020 muestran una tendencia al alza y reportan un aumento de $22,9 \%$ del PIB en el año 2013 a $24,4 \%$ del PIB en el año 2014, y proyectan alcanzar 27\% del PIB al año 2020 (9). La recaudación tributaria aumentó de
15,7\% del PIB en el 2013 a 18,3\%, pero las contribuciones sociales se mantuvieron en $3,2 \%$ del PIB.

Honduras tiene una estructura general de impuestos directos e indirectos que tiende a ser regresiva $(21,24)$. La participación de impuestos directos, que suelen ser progresivos, aumentó en 10 pp.

El impuesto al tabaco y a las bebidas alcohólicas también se ajustó al alza en la reforma de 2014, pero los ingresos asociados a la cerveza, aguardiente, licor, gaseosas y cigarrillos cayeron a 0,473\% del PIB en el año 2015.

Se considera crear un impuesto nuevo a las comidas procesadas marcado para salud, asumiendo que la demanda por comidas procesadas se comportaría igual que la de gaseosas y que el impuesto (tasa o monto por kilo) sería tal que genere la misma recaudación que el impuesto a las gaseosas. De esta manera, la proyección de los ingresos aplicado a las gaseosas muestra que en el período 2016-2020 se podría acumular 1,001 pp del PIB. La proyección con una tasa $10 \%$ más alta se podría generar 1,073 pp del PIB, solo 0,072 pp adicionales. Si los ingresos de los otros impuestos al pecado, que fueron aumentados en 2014, se marcaran para el sector salud, esto podría llevar a acumular 4,118 pp del PIB o 0,686 pp al año.

Esta fuente tiene una perspectiva política positiva, aunque por el lado de ampliar la base tributaria de los impuestos directos, reducir exoneraciones y disminuir la evasión fiscal. La creación de impuestos específicos a comidas procesadas y gaseosas también tendría aceptación, pero no así otros impuestos.

Los encuestados opinaron que era casi imposible o imposible el incremento de la tasa de algún impuesto, aunque 55\% considera factible incrementar la base de algún impuesto. El 73\% rechaza o ve poco factible la creación de un nuevo impuesto, pero la opción de un nuevo impuesto a las comidas procesadas fue considerada como factible por $45 \%$ de ellos. Destinar los ingresos de impuestos al pecado al sector salud también generó opiniones favorables (82\%), sobre todo porque se considera el daño que estos causan a la salud de la población.

\section{Nuevos ingresos: contribuciones sociales a salud}

Este análisis considera el hecho que las contribuciones al IHSS serán una fuente de espacio fiscal solo si la reforma 
Cuadro 2. Escenarios para las aportaciones a IHSS, 2015-2020

\begin{tabular}{|c|c|c|c|c|c|c|c|c|}
\hline \multirow{2}{*}{ Escenario } & \multirow{2}{*}{ Cambio \% cotizantes } & \multicolumn{7}{|c|}{ Porcentaje del PIB por año } \\
\hline & & 2015 & 2016 & 2017 & 2018 & 2019 & 2020 & Acumulado 2015-2020 \\
\hline 1. Sin gradualidad en el techo & 2,7 & 2,46 & 2,44 & 2,42 & 2,39 & 2,36 & 2,33 & 14,40 \\
\hline 2. Con gradualidad en el techo & 2,7 & 2,59 & 2,69 & 2,80 & 2,91 & 3,01 & 3,12 & 17,12 \\
\hline Diferencia con 1 & & 0,12 & 0,25 & 0,38 & 0,52 & 0,65 & 0,79 & 2,71 \\
\hline 3. Aumento en formalización & 4,1 & 2,62 & 2,73 & 2,84 & 2,95 & 3,05 & 3,17 & 17,36 \\
\hline Diferencia con 2 & & 0,04 & 0,04 & 0,04 & 0,04 & 0,04 & 0,04 & 0,24 \\
\hline 4. Para lograr 1 pto del PIB & 8,7 & 2,74 & 2,85 & 2,96 & 3,08 & 3,19 & 3,30 & 18,12 \\
\hline Diferencia con 2 & & 0,15 & 0,16 & 0,16 & 0,17 & 0,18 & 0,18 & 1,00 \\
\hline
\end{tabular}

Elaboración propia.

IHSS, Instituto Hondureño de Seguridad Social; PIB, producto interno bruto.

sanitaria considera la mancomunación solidaria de recursos ya que, en la actualidad, el del IHSS es un fondo cerrado y estanco que no aumenta los recursos para el subsector SESAL. En cualquier caso, su aumento sí podría generar ahorros para SESAL.

En el escenario base, la recaudación disminuiría a 2,33\% del PIB en el año 2020 (cuadro 2), porque se mantiene un techo a la base de la contribución. En el segundo escenario, se obtendría 3,12\% del PIB en el 2020. El tercer escenario, que aumenta la formalización del mercado laboral, solo generaría 3,17\% del PIB en el 2020, o 0,05 pp adicionales. Los escenarios muestran que, aún con una formalización acelerada, el techo de la contribución limita el aumento de ingresos por esta vía.

Desde la factibilidad política, la aceleración de la formalización se ve lejana, ya que requeriría de una política multisectorial no contemplada. Asimismo, 73\% de los encuestados considera que sería poco factible o imposible aumentar las contribuciones al IHSS.

\section{Eficiencia}

La creación de un régimen de piso de protección social con el Fondo de Solidaridad y Protección Social para la Reducción de la Pobreza busca la eficiencia en el gasto público con el manejo integrado de los recursos. La implementación de redes integradas de salud podría reducir la necesidad de inversiones y expandir el acceso, sobre todo hacia el primer nivel de atención. Si bien no se encontraron estimaciones de ganancias por eficiencia, un estudio recomienda que los esfuerzos deberían centrarse en reducir más las ineficiencias en el gasto corriente, mejorar la gestión de medicamentos y recursos humanos, y fortalecer los mecanismos de rendición de cuentas (22).

El apoyo político de esta fuente se ha establecido en los acuerdos firmados con el Fondo Moentario Internacional (FMI). En el año 2013, se dio un primer paso para mejorar la eficiencia al establecer gabinetes sectoriales.

\section{Repriorización del gasto}

El GPS como porcentaje del gasto total del gobierno muestra grandes variaciones en el tiempo, en parte porque la participación de la ayuda externa desplazó parte del gasto en este sector. La posibilidad de variar las participaciones de los sectores en el presupuesto público está sujeta a la rigidez que se da por el gasto en personal, el que suele ser, desde un punto de vista político, difícil de modificar en el corto y mediano plazos. Según Escobar (21), entre los años 2004 y 2008, más de la mitad del gasto corriente del gobierno de Honduras estuvo orientado al gasto en personal y absorbió la disminución del gasto en intereses.

La repriorización del gasto en salud a través de recortes en otros sectores sería difícil de implementar, ya que podría traducirse en retrocesos en otras áreas. Sin embargo, políticamente se ve más factible si se hace a través de la asignación de nuevos ingresos al sector salud, en el aumento presupuestario global.

\section{Ayuda externa}

La ayuda externa no es una fuente recomendada porque son decisiones que no dependen del país receptor y porque, desde una perspectiva histórica, son poco predecibles $(11,21)$ y que podrían reducir la participación de salud en el presupuesto público (crowding out) (21).
Solo se debería considerar si no genera dicho desplazamiento.

La ayuda externa tiene apoyo de $81 \%$ de los encuestados que la considera como muy factible y factible.

\section{Deuda}

De la misma manera que en el caso anterior, tampoco se recomienda adquirir créditos $(11,21)$, ya que genera requerimientos futuros del pago de la deuda. Honduras es un país que ha tenido históricamente un alto nivel de endeudamiento público. Después de lograr una disminución importante en su deuda pública bruta entre el año 2003 y 2008, esta ha vuelto a aumentar y recién se revertirá a partir del año 2018 (9).

En un contexto posreforma tributaria, esta fuente solo debería implementarse en la medida que financie inversiones que puedan generar ingresos futuros para el pago de la deuda (autofinanciable). La retracción de las deudas ya es uno de los acuerdos con el FMI. Sin embargo, entre los encuestados, $82 \%$ considera poco factible o imposible aumentar la deuda a través de fuentes internas, en cambio $73 \%$ piensa que sería factible a través de fuentes externas.

\section{DISCUSIÓN}

En el cuadro 3 se presenta un resumen de los resultados. El caso de Honduras es especial porque se realiza el estudio con una reforma tributaria reciente y con varios acuerdos de reforma del sector público. Dichas reformas se hicieron sin considerar los compromisos que se establecerían con las reformas que se están emprendiendo específicamente en el sector salud. Aunque la literatura muestra que se puede identificar fuentes de EF, en el caso de Honduras 


\section{Cuadro 3. Resumen de análisis y estimaciones}

\begin{tabular}{|c|c|c|}
\hline Fuente & Factibilidad & $\triangle$ GPS pp PIB promedio \\
\hline $\begin{array}{l}\text { Condición básica: } \\
\text { crecimiento económico }\end{array}$ & - Genera pocos recursos y por lo tanto es una señal que se requieren de otras fuentes. & $\begin{array}{l}\text { - } \quad \text { Pesimista: }-0,0090 \\
\text { - } \quad \text { Neutro: } 0,0133 \\
\text { - Optimista: } 0,0351\end{array}$ \\
\hline Recaudación tributaria & $\begin{array}{l}\text { - La reforma tributaria del año } 2014 \text { es muy reciente. } \\
\text { - Existe disposición a asignar los recursos de impuestos al pecado al sector salud. }\end{array}$ & $\begin{array}{l}\text { - Aguas azucaradas: 0,167 } \\
\text { - Comidas procesadas: 0,167 }\end{array}$ \\
\hline Contribuciones sociales & $\begin{array}{l}\text { - El techo en la base contributiva limita la recaudación, aún con una formalización más acelerada. } \\
\text { - } \quad \text { Lo parece haber el esfuerzo necesario para acelerar la formalización. } \\
\text { demanda por servicios de SESAL }\end{array}$ & $\begin{array}{l}\text { - Gradualidad: 0,452 pp } \\
\text { - Formalización: 0,492 pp }\end{array}$ \\
\hline $\begin{array}{l}\text { Eficiencia del gasto } \\
\text { público }\end{array}$ & $\begin{array}{l}\text { - Medidas como: implementar redes integradas de salud, reducir el gasto corriente, mejorar la gestión de } \\
\text { medicamentos y recursos humanos } \\
\text { - Se debe aprovechar la reforma que está en proceso para obtener recursos a través de medidas que mejoren } \\
\text { la eficiencia }\end{array}$ & - ND \\
\hline Repriorización del gasto & - No se perfila como una fuente factible & - ND \\
\hline Ayuda externa y crédito & $\begin{array}{l}\text { - Existe interés de utilizar estas fuentes, pero se deben considerar el desplazamiento histórico de la ayuda } \\
\text { externa al gasto público y las obligaciones de pagos futuros de la deuda. }\end{array}$ & - ND \\
\hline
\end{tabular}

Elaboración propia.

SESAL, Secretaría de Salud; IHSS; Instituto Hondureño de Seguridad Social; GPS, gasto público en salud; PIB, producto interno bruto; ND, no disponible; pp, puntos porcentuales.

este se ve limitado por los compromisos recientes $(5,11,21)$.

Sin embargo, el largo proceso de formulación y amplia participación en importantes documentos dieron paso a la creación de una "imagen o visión país" que ha permitido emprender las reformas sectoriales y que han impulsado un clima de confianza en Honduras (25). Esto también ha permitido implementar la reorganización de la gestión pública, con asignación de responsabilidades y rendición de cuentas. En este sentido, como aún no existe una estimación de los requerimientos financieros para la implementación de la reforma en el sector salud, en parte porque no se ha terminado de diseñar la reforma, es importante que las decisiones sobre su alcance se basen en la capacidad del gobierno de generar los recursos necesarios para financiarla.

Honduras tiene un GPS de 4,3\% del $\mathrm{PIB}$, lo que implica que necesitaría 1,7 pp adicionales para cumplir con la meta de 6,0\% del PIB. Las últimas reformas muestran que Honduras está encaminada para lograr la condición básica de crecimiento económico y estabilidad. Sin embargo, dicho crecimiento no genera mayores recursos para SESAL, aunque sí lo podría lograr para IHSS. Sin medidas adicionales orientadas a crear EF y sin mancomunación solidaria de recursos públicos, el GPS muestra una tendencia a la baja que aumentaría la brecha.

La generación de nuevos ingresos - a través de los ingresos tributarios o de las contribuciones sociales en salud- se ve limitada porque Honduras acaba de implementar una reforma tributaria (2014) y también han modificado las contribuciones al IHSS que incluye una gradualidad en el aumento del techo (2015) (26, 27). Las fuentes más prometedoras son los ingresos por impuesto a las bebidas azucaradas y a la creación de un nuevo impuesto a las comidas procesadas, y que estos ingresos se asignen de forma exclusiva para el sector salud. Esto generaría 0,167 pp del PIB al año con el impuesto actual o 0,179 pp si se considera el aumento de $10 \%$. La justificación para asignar la recaudación a este impuesto es que las consecuencias del sobreconsumo las asume el sector salud a través de la atención de las enfermedades que causan estos productos.

Los cambios que se están implementando en las contribuciones sociales, en particular la gradualidad en el aumento del techo de la base contributiva, generaría un estimado de nuevos recursos de 0,45 pp adicionales al año, asumiendo el mismo nivel de crecimiento en los cotizantes observado en los últimos 5 años. Las políticas orientadas a acelerar la formalización laboral serían una alternativa a explorar. Esto genera más recursos para IHSS y más asegurados, que podría reducir la presión sobre los recursos de SESAL. Cabe destacar, sin embargo, que el techo de la base contributiva está por debajo del salario mínimo. El salario mínimo promedio en el 2015 fue de HNL 7 850, mientras que el techo fue de HNL 7350 (27, 28). Así, la contribución es regresiva, donde las personas que ganan más pagan proporcionalmente menos que los que ganan menos, por lo que la sostenibilidad financiera del IHSS podría ponerse en riesgo en la medida que no se revise sus requerimientos con estudios actuariales.

Como se mencionó, el aumento de cotizantes al IHSS tiene dos implicancias para el gobierno y SESAL: por un lado, si los nuevos cotizantes son del sector público, el gobierno deberá aportar más a IHSS, lo que representa un aumento de sus requerimientos financieros. Por otro lado, se liberaría parte de la presión en la demanda por servicios de SESAL, lo que podría entenderse como ahorros en el gasto de SESAL en servicios de salud. Si la diferencia es positiva, el compromiso del gobierno con las cotizaciones a IHSS es menor que el ahorro por el cambio en la demanda, SESAL podría contar con más recursos.

La reforma de la política fiscal contempla mejorar la eficiencia de la administración pública. No se contó con estimaciones sobre el posible impacto que esto tendría en los recursos financieros. Sin embargo, existen diferentes medidas que el mismo sector salud podría implementar para hacer un mejor uso de los recursos que tiene disponible. Mejorar la eficiencia del gasto siempre tiene apoyo político.

La repriorización del sector salud es quizás una de las fuentes más prometedoras. Ya existe una política nacional y la aprobación de las leyes antes mencionadas que le dan sustento al hecho de que la salud es una prioridad. Esta política se podría traducir en EF para la salud en la medida que la generación de nuevos recursos se asigne dándole la prioridad al sector salud. Sin embargo, es importante 
que las reformas se planteen en el marco de la disponibilidad de recursos para implementarlas para que estas tengan la oportunidad de cumplir con el compromiso de mejorar el acceso a los servicios de salud.

La ayuda externa y el aumento de la deuda pública no se perfilan como fuentes para el espacio fiscal en la medida que estas dos fuentes están resguardadas por los acuerdos establecidos por el gobierno con organismos como el FMI. mación de los requerimientos financieros para el sector salud y su reforma, de tal manera que se toma como referencia el
Una limitación es que no existe una esti-

acuerdo de la OPS de alcanzar un GPS de $6 \%$ del PIB (10).

\section{CONCLUSIONES}

Para acompañar los esfuerzos de reforma y alcanzar la meta de un GPS de $6 \%$ del PIB, Honduras deberá generar espacio fiscal. La reciente reforma tributaria pone limitaciones para generar nuevos recursos, pero existen alternativas razonables a través de impuestos al pecado, la repriorización del gasto $\mathrm{y}$ también de una revisión del techo a la base contributiva del IHSS si se considerara la mancomunación de los recursos

\section{REFERENCIAS}

1. Banco Mundial. Indicadores del Desarrollo Mundial. Banco Mundial, 2016. Disponible en: http://databank.worldbank.org/data/ reports.aspx? source $=$ world-development-indicators Acceso en abril de 2016.

2. Gobierno de la República de Honduras. Hacia la salud universal: logros, avances y desafíos del sector salud de Honduras. Tegucigalpa: Gobierno de la República de Honduras, 2016.

3. Tandon A, Cashin C. Assessing public expenditure on health from a fiscal space perspective. Washington D.C.: World Bank; 2010.

4. Gordon R, Li W. Tax structures in developing countries: Many puzzles and a possible explanation. J Public Econ. 2009;93(7): 855-66.

5. Durán-Valverde F, Pacheco JF. Fiscal space and the extension of social protection: Lessons learnt from developing countries: Bolivia, Botswana, Brazil, Costa Rica, Lesotho, Namibia, Thailand and South Africa. ESS. 2012;33.

6. Rao MG, Seth A. Fiscal Space for achieving the millennium development goals and implementing the tenth plan in Bhutan. Econ Polit Weekly. 2009;44(35):51-9.

7. Organización Mundial de la Salud (OMS). Base de datos Global de Gasto en Salud (Global Health Expenditure Database). Organización Mundial de la Salud, 2016. Disponible en: http://apps.who.int/nha/ database/Select/Indicators/en Acceso en abril de 2016.

8. Fondo Monetario Internacional (FMI). Honduras: Request for a stand-by arrangement and an arrangement under the standby credit facility - Staff Report; Press Release. Washington, D.C.: FMI, 2014.

9. Fondo Monetario Internacional (FMI). World Economic Outlook. Fondo Monetario Internacional, 2016. Disponible en: https:/ / www.imf.org/external/pubs / $\mathrm{ft} /$ weo/2015/02/weodata/index.aspx Acceso en abril de 2016.

10. Organización Panamericana de la Salud/ Organización Mundial de la Salud (OPS/
OMS). 53o Consejo Directivo: 66a. Sesión del Comité Regional de la OMS para las Américas. CD53/FR. 66 ed. Washington, D.C.: OPS; 2014.

11. Heller PS. The prospects of creating 'fiscal space' for the health sector. Health Policy Plann. 2006;21(2):75-9.

12. Heller PS. Understanding fiscal space. International Monetary Fund; 2005.

13. Sharma J. An assessment of fiscal space for health in Bhutan. Int J Health Plan M. 2016;31(3):296-308.

14. Matus-López M, Prieto Toledo L, Cid Pedraza C. Evaluación del espacio fiscal para la salud en Perú. Rev Panam Salud Publica. 2016;40(1):64-9.

15. Secretaría de Finanzas de Honduras (SEFIN). Informe de la liquidación del presupuesto general de ingresos y egresos de la República: ejercicio fiscal 2010. Tegucigalpa: SEFIN, 2011.

16. Secretaría de Finanzas de Honduras (SEFIN). Informe de la liquidación del presupuesto general de ingresos y egresos de la República: ejercicio fiscal 2011. Tegucigalpa: SEFIN, 2012.

17. Secretaría de Finanzas de Honduras (SEFIN). Informe de la liquidación del presupuesto general de ingresos y egresos de la República y de las instituciones descentralizadas: ejercicio fiscal 2012. Tegucigalpa: SEFIN, 2013.

18. Secretaría de Finanzas de Honduras (SEFIN). Informe de la liquidación del presupuesto general de ingresos y egresos de la República y de las instituciones descentralizadas: ejercicio fiscal 2013. Tegucigalpa: SEFIN, 2014.

19. Secretaría de Finanzas de Honduras. Informe de la liquidación del presupuesto general de ingresos y egresos de la administración pública: ejercicio fiscal 2014. Tegucigalpa: SEFIN, 2015.

20. Instituto Hondureño de Seguridad Social Tegucigalpa: IHSS, Estadística Dd; 2016.

21. Escobar LE. Honduras: espacio fiscal para la inversión social y productiva. (IHSS). IHSS en Cifras Serie 2004-2015. públicos. Un riesgo importante es que la reforma en el sector salud, que aún se está formulando, no genere necesidades financieras fuera de las posibilidades del gobierno que, en última instancia, reduzcan la posibilidad de éxito de su implementación.

Conflicto de intereses. Ninguno declarado por los autores.

Declaración. Las opiniones expresadas en este manuscrito son responsabilidad de los autores y no reflejan necesariamente los criterios ni la política de la RPSP/PAJPH y /o de la OPS.
Tegucigalpa, Honduras: Programa de las Naciones Unidas para el DesarrolloHonduras; 2010; 40 p.

22. Banco Mundial. Honduras: estudio de gasto público social y sus instituciones: educación, salud, protección social y empleo. Washington, D.C.: Grupo Banco Mundial, 2015.

23. Fondo Monetario Internacional (FMI). Government Finance Statistics Yearbook. Washington, D.C.: FMI, 2016. Disponible en: http: / / data.imf.org/?sk=a0867067-d23c4ebc-ad23-d3b015045405\&sId=143569791 4186 Acceso en marzo de 2016.

24. Cubero R, Hollar IV. Equity and fiscal policy: the income distribution effects of taxation and social spending in Central America. IMF Working Papers. 2010: $1-42$.

25. Banco Mundial. Honduras: informe sobre el gasto público. Washington, D.C.: Banco Mundial, 2007.

26. Poder Legislativo de Honduras. Reforma del Código Tributario. Decreto No. 3932013. Publicado en La Gaceta, No. 33.369 (2013).

27. Secretaría de Trabajo y Seguridad Social de Honduras. Gradualidad de las contribuciones de empleadores y trabajadores para financiar los diferentes regímenes y pilares que constituyen el Sistema de Protección Social. Acuerdo No. STSS-390 2015. Publicado en La Gaceta, No. 33.879 (2015).

28. Secretaría de Trabajo y Seguridad Social de Honduras. Salario mínimo 2014-16. Acuerdo No. STSS-599-2013. Publicado en La Gaceta, No. 33.313 (2013).

Manuscrito recibido el 1 de junio de 2017. Aceptado para publicación, tras revisión, el 22 de agosto de 2017. 
ABSTRACT Objective. To analyze sources of fiscal space for the health sector in Honduras, in the context of sectoral reform, with a commitment to achieving the target of public expenditure on health equivalent to $6 \%$ of gross domestic product (GDP).

Fiscal space for health in Honduras

Keywords Healthcare financing; universal coverage; Latin America; Honduras. ted on the basis of a literature review and secondary data. The size of each source was estimated from official data, international statistics, and previous studies. In parallel to this study, political feasibility was analyzed and an online survey was administered to key actors. sources must be identified in order to generate new resources. The recent tax reform limits the political feasibility of creating new taxes, except for "sin taxes", that could be measures that could make resources available by improving efficiency in the sector. market. ure on health equivalent to $6 \%$ of GDP with the support of plans for sectoral reform, but its options are limited by the recent tax reform. The reform of social protection in mentation of the reform.

RESUMO Objetivo. Analisar as fontes de espaço fiscal para saúde em Honduras no contexto da reforma do setor, com o compromisso de alcançar uma meta de gasto público em saúde de $6 \%$ do produto interno bruto (PIB).

\section{Espaço fiscal para saúde em Honduras}

An analysis of baseline conditions and sources of fiscal space was conduc-

Results. Estimates of baseline conditions for economic growth show that other used exclusively to fund health. Social protection reform paves the way to explore One limitation on public expenditure based on social security contributions is the ceiling on taxable income, notwithstanding acceleration in the formalization of the labor

Conclusions. Honduras can advance towards achieving the target of public expendithealth should consider additional available resources so as not to jeopardize imple-

Métodos. Foi realizada uma análise da situação básica e das fontes de espaço fiscal com base em uma revisão da literatura e dados secundários. Foi estimado o tamanho das fontes com dados oficiais, estatísticas internacionais e estudos prévios. Para complementar o estudo, foi realizada uma análise da viabilidade política e uma pesquisa online com os principais interessados diretos.

Resultados. As estimativas da situação básica de crescimento econômico indicam que é necessário identificar novas fontes para geração de recursos. A reforma tributária recente restringe a viabilidade política de criar impostos, exceto impostos pecuniários cuja arrecadação poderia ser alocada exclusivamente à saúde. A reforma da previdência social abre caminho para examinar medidas para liberar recursos com a melhoria da eficiência do setor. Uma limitante no caso do gasto público proveniente da previdência social é o teto da base contributiva, independente de uma aceleração na formalização das relações de trabalho.

Conclusões. Honduras pode procurar alcançar uma meta de gasto público em saúde de $6 \%$ do PIB que respalde os planos de reforma setorial, mas as opções são limitadas pela recente reforma tributária. A reforma da previdência social em saúde deve considerar os recursos adicionais que terá à disposição para não comprometer a própria implementação.

Palavras-chave Financiamento da assistência à saúde; cobertura universal; América Latina; Honduras. 